\title{
COMPLEX GEOPHYSICAL PROSPECTION - ELECTRICAL RESISTIVITY TOMOGRAPHY AND GEOMAGNETIC SURVEY FOR DETECTING LIMESTONE INCLUSIONS IN MINI MARITSA IZTOK EAD
}

DOI: http://dx.doi.org/10.18509/GBP.2017.05

UDC: 552.541:550.832(497.2)

\author{
Maya Grigorova \\ Christian Tzankov \\ Atanas Kisyov \\ Ivaylo Koprev \\ Stefan Dimovski \\ University of Mining and Geology “St. Ivan Rilski”, Bulgaria
}

\begin{abstract}
The presence of consolidated limestone inclusions in Mini Maritsa Iztok EAD has great influence on the pace of productivity and becomes an issue of primary importance. Complex geophysical prospections were performed in order to collect information about the presence, location and characteristics of the solid limestone bodies incorporated in the mostly clayey medium. The use of selected high-resolution geophysical methods electrical resistivity tomography (ERT) and geomagnetic survey proved to be very effective in the non-destructive survey of the investigated area.
\end{abstract}

Keywords: electrical resistivity tomography (ERT), geomagnetic survey, electromagnetic field, limestone inclusions.

\section{INTRODUCTION}

Mini Maritsa Iztok EAD is the largest coal mining company in the Republic of Bulgaria. It works the Maritsa Iztok lignite field. Coal seams in the Maritsa Iztok basin are located relatively low at $6-10 \mathrm{~m}$ to $110-120 \mathrm{~m}$ below the surface. The total thickness of the coalfield complex is around $35-40 \mathrm{~m}$ and is divided by clay layers into three seams. The medium (second) seam is the main coal carrier with an average thickness of $15-25 \mathrm{~m}$. The deposit is operated by three opencast mines - Troyanovo-1, Troyanovo-North and Troyanovo-3. The present study helps in understanding the geological conditions in opencast Troyanovo-3. Troyanovo-3 mine is located in the southern part of the Maritsa Iztok Basin and is in the form of a rectangle with west-east orientation, a length of 12 kilometers and a width of about 2,5-3 $\mathrm{km}$. The payable reserves are calculated at $336,230,000$ tonnes. The coal layer is located at an average depth of 70-80 m. Extraction conditions in the opencast Troyanovo-3 become harder because of presence of solid limestone inclusions. These solid limestones bodies make extraction operations extremely challenging.

This study is focusing on opencast coal exploration in the area of Mini Maritsa Iztok EAD. The usefulness of geophysical methods in mapping the hard rock inclusions in the overburden of the lignite mines is outlined. 


\section{DESCRIPTION OF THE TECHNOLOGY}

Geophysical methods can assist in solving the problem through the detection of different physical properties of the soil, by sending a physical signal and receiving it again (such as current, sonic wave, EM wave, etc.). Electrical Resistivity Tomography (ERT) is a technique that can detect and characterize layers by exploiting resistivity contrasts between different layers using electrical current. The aim of the electrical resistivity tomography (ERT) technique is to scan the subsurface along the survey line using a selected electrode array [1], [2].

It is an advanced geophysical method used to determine the subsurface resistivity distribution by making measurements on the ground surface. ERT data are rapidly collected with an automated multi-electrode resistivity meter - Terrameter SAS 1000.

The acquisition of resistivity data involves the injection of current into the ground using a pair of electrodes and then the resulting potential field is measured by a corresponding pair of potential electrodes. The field set-up requires the deployment of an array of regularly spaced electrodes, which are connected to a central control unit via multi-core cables. Resistivity data are then recorded through complex combinations of current and potential electrode pairs to build up a pseudo cross-section of apparent resistivity beneath the survey line. The depth of investigation depends on the electrode separation and geometry, with greater electrode separations yielding resistivity measurements from greater depths.

For example, Figure 1 shows a possible sequence of measurements for the Wenner electrode array for a system with 20 electrodes.

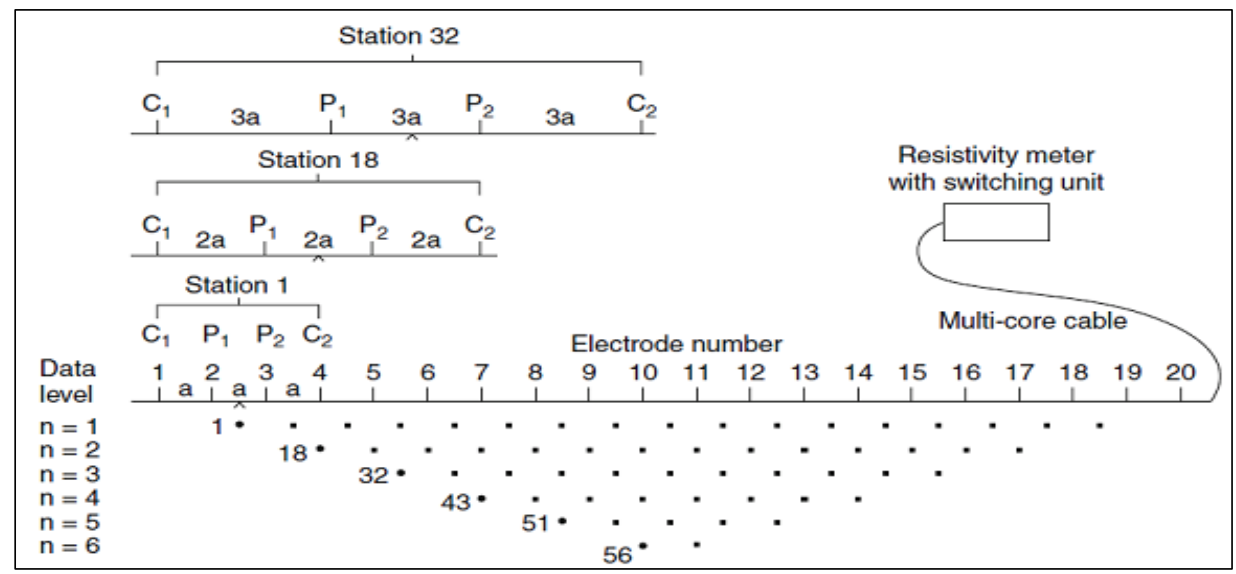

Figure 1. The main components and arrangement of electrodes for a 2D electrical survey (ERT) Wenner array

In this example, the spacing between adjacent electrodes is "a". The first measurement is made by using two current electrode $\mathrm{C} 1 \& \mathrm{C} 2$, and two potential electrodes P1 \& P2. These are done in the electrode positions 1, 2, 3, 4 the measured point depth is about $20 \%$ - $25 \%$ of the total length (3a). When using electrode positions $2,3,4,5$ and so on to the final position $17,18,19,20$, then it is finished the first depth of measurements at level $\mathrm{n}=1$ (Figure 1). The measurements are then repeated but electrode separation is increased to increase the depth level. For example if we increased separation between electrodes into " $2 \mathrm{a}$ ", the second row of measurements at $\mathrm{n}=2$ is measured. The process is repeated until we get resistivity values for the whole image (pseudosection) and all desired depths from depth level $n=1$ to $n=6$ [3]. 
Information about the location, depth, size and extent of buried limestone inclusions may be determined by means of geophysical investigation, which is carried out easily and quickly on the surface [4]. The geophysical methods can locate them by measuring their magnetic susceptibility, electrical resistivity or conductivity [5].

The aim of conducted magnetic survey is to investigate subsurface geology on the basis of the anomalies in the earth's magnetic field resulting from the magnetic properties of the underlying rocks. In general, the magnetic content (susceptibility) of rocks is extremely variable depending on the type of rock and the environment it is in. Sedimentary rocks generally have a very small magnetic susceptibility compared with igneous or metamorphic rocks. Most magnetic surveys are designed to map the geologic structure on or inside the basement rocks. Ground magnetic measurements do provide more detailed information on sub-surface structures. The magnetic data are collected with two magnetometers designed by GEM Systems, Inc., Canada. The first one is magnetometer model Proton GSM-19TGW for field measurements in walking gradientometer mode and the second one is magnetometer model Overhauser GSM-19 acting as a base station in order to measure the daily variations of the magnetic field.

\section{FIELD MEASUREMENTS AND DATA PROCESSING}

In this study ERT is used along with magnetic survey to describe the presence and location of the solid limestone inclusions in the field section. The precise location of the geophysical surveying lines in the area of Mini Maritsa Iztok EAD is illustrated in Figure 2. The ERT field measurements were performed along 7 profiles (six of them with a length of $92 \mathrm{~m}$, and the ERT -7 with a length of $115 \mathrm{~m}$ ) having a total length of $667 \mathrm{~m}$.

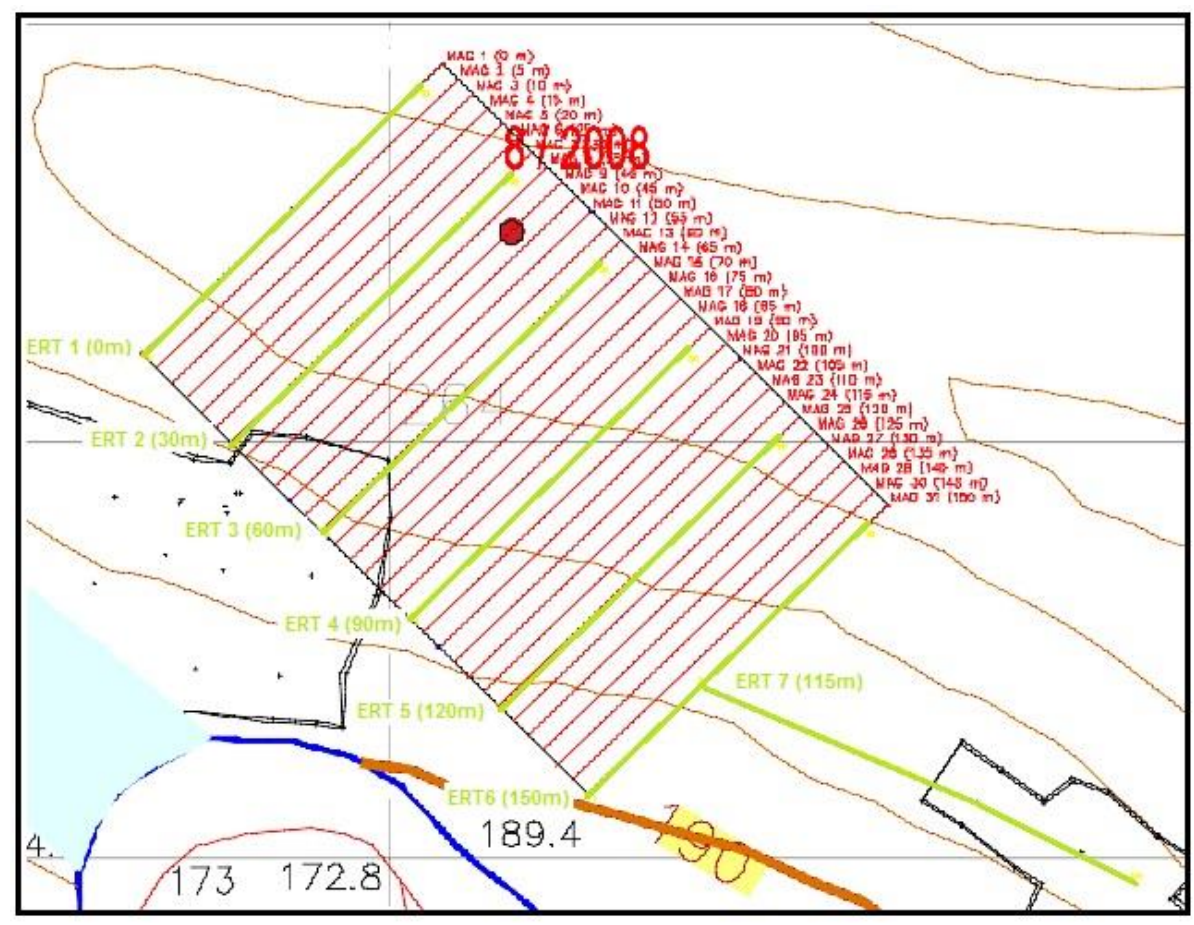

Figure 2. Field measurements situation plan

The geomagnetic field measurements were performed along 32 profiles with a length of $100 \mathrm{~m}$ having a total length of $3200 \mathrm{~m}$. Data acquisition for both surveys involves taking 
a series of points at regular intervals on a survey grid. The spacing between grid lines for ERT sections is $30 \mathrm{~m}$ and for magnetic survey it is $5 \mathrm{~m}$.

Data processing is based on an iterative routine involving determination of a twodimensional (2D) model of the subsurface, which is then compared to the observed data and revised. ERT data processing and modeling were done using the RES2DINV. This is a computer program that automatically determines the $2 \mathrm{D}$ resistivity model for the data obtained [6]. The program makes inversion by dividing the original data into rectangular bocks. The program uses the forward modeling to calculate the apparent resistivity value [7]. The results from such surveys are plotted in the form of a pseudosection which gives an approximate picture of the subsurface geology.

Magnetometer data are stored digitally by the survey instrument and downloaded to a field computer at the end of the day. The magnetic data are then processed to enhance any identifiable anomalies using interactive software (Encom PA) and then presented on colour-contoured plots.

\section{RESULTS AND INTERPRETATION}

The results illustrate the potential of electrical resistivity and geomagnetic methods to separate different layers and monitor the subsurface location and size of limestone inclusions based on electrical resistivity and magnetic susceptibility of rocks.

The true resistivity models are presented as colour contour sections revealing spatial variation in subsurface resistivity. The analysis of the ERT lines allows to be made the following important conclusions:

1. Based on the electrical resistivity measured the soil show no homogeneous effects;

2. Average electrical resistivity of the medium is between $10-50 \Omega \mathrm{m}$;

3. ERT profiles can be separated into four electrical resistivity zones (Figure 3):

- Under the surface zone, the electrical resistivity values decrease to $10-15 \Omega \mathrm{m}$ and the geoelectrical boundary is well distinguished. This section is defined as first electrical resistivity zone and is consider as composed of mostly silty clay and random limestone fragments.

- Second electrical resistivity zone is consider as a zone of relatively higher resistivity values (up to about $30-35 \Omega \mathrm{m}$ ). According to the section this zone indicates presence of limestone concretions in the clayed medium.

- Third electrical resistivity zone is composed of silty clay mixed with sand in some places and has average resistivity between $35-50 \Omega \mathrm{m}$. This zone shows different resistivity values in the left and right parts. This is most probably due to the change in soil composition - from silty or clay into more sand component.

- The fourth electrical resistivity zone shows relatively high resistivity value between $15-35 \Omega \mathrm{m}$. This is probably due to the presence of limestone inclusions in the clayed depositions in the surface zone of the sections. 


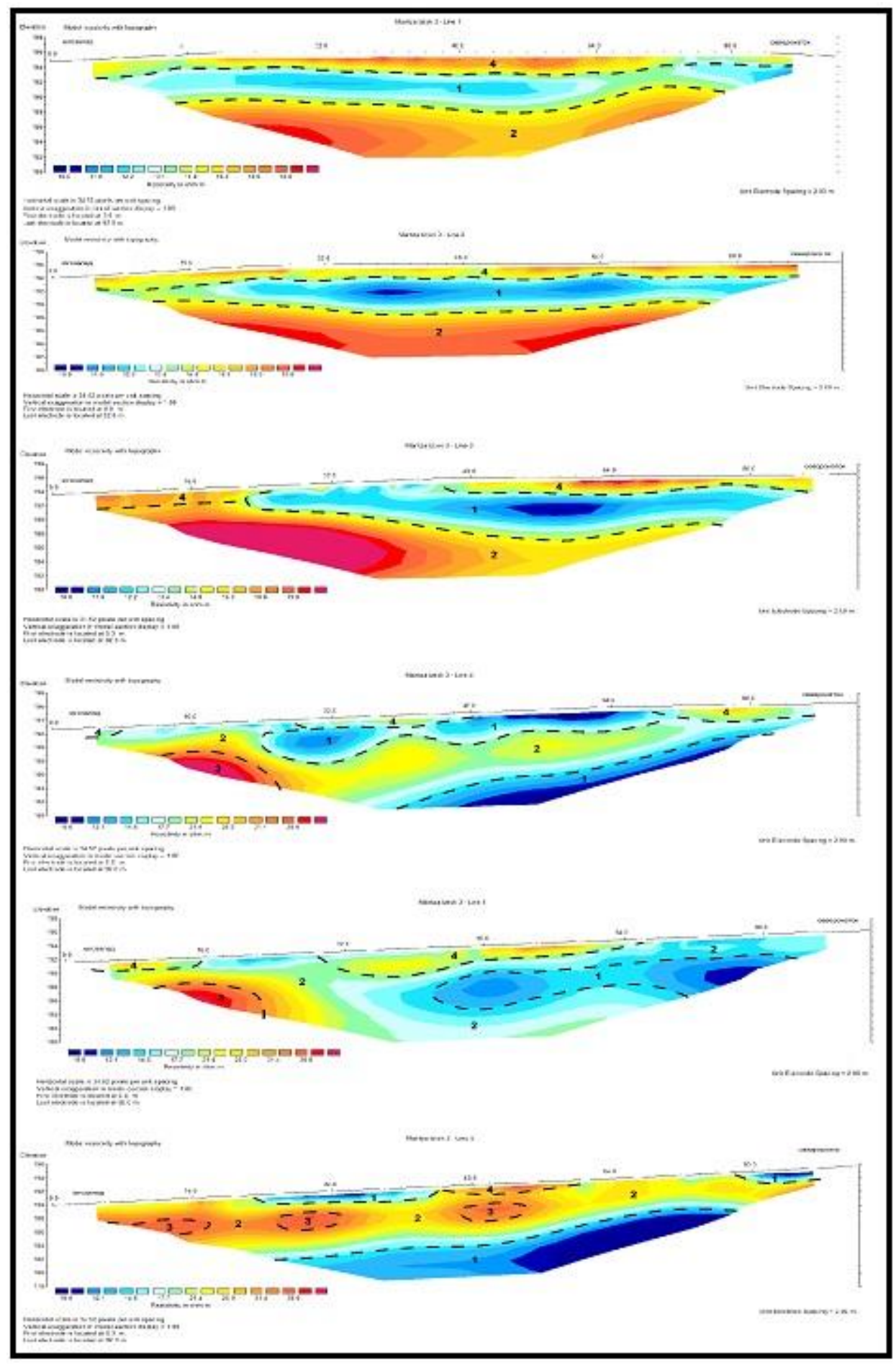

Figure 3. ERT lines from 1 to 6 .

Line ERT-7 (Figure 4) is located almost perpendicular to the main measurement profiles, east of the study area. The aim of this additional profile is to get additional information about the observed area. 


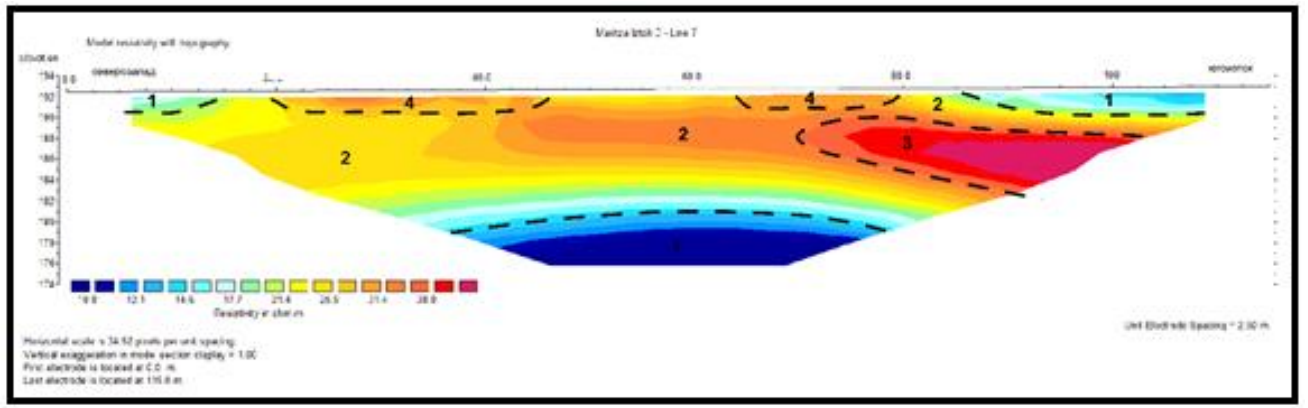

Figure 4. Line ERT-7.

The results of the magnetic survey are usually presented as total field plots. The total field data may be used to observe the general character of the magnetic field across the survey area. Various interpretation techniques are applied to the data using interactive software (Encom PA) to identify the targeted anomalies. A combination of contouring and colour shading is used to highlight anomaly patterns. Survey results are presented as plans tied in to site coordinates (Figure 5).

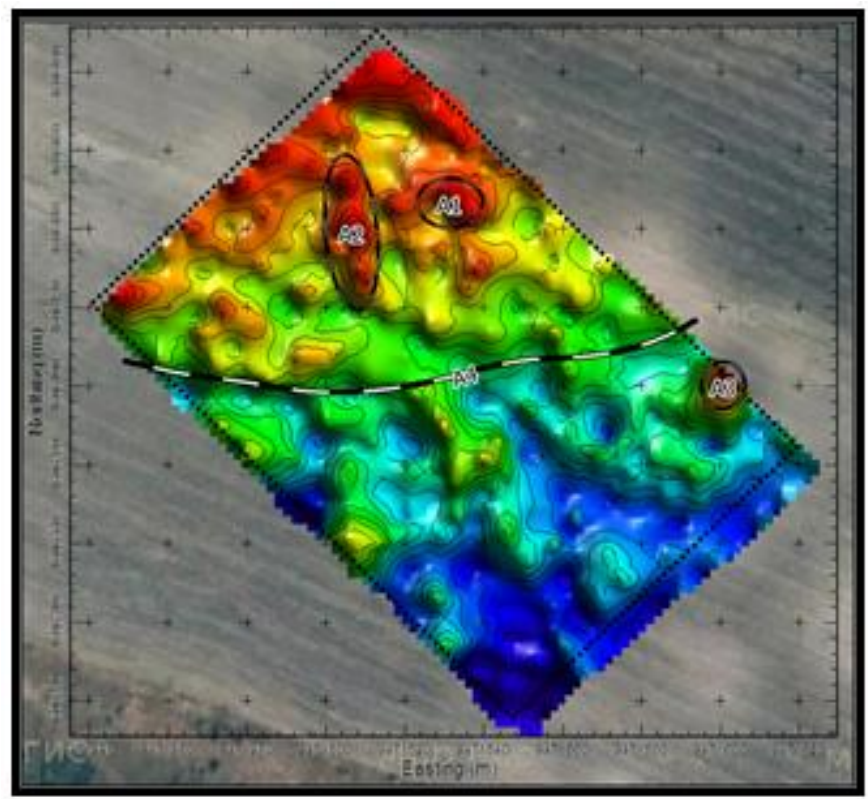

Figure 5. Location of geomagnetic data defined anomalous zones imposed on a map of local anomalies of the geomagnetic field

The analysis of the magnetic survey results allows to be made the following important conclusions:

$>$ There are several anomaly zones (A1, A2, A3), due to the presence of ferrous elements in the research area like wells casings or other objects which tend to have a higher magnetic properties;

Anomalous zone A4 is linear and it is the boundary between two large sections. North of the line of A4 the anomalous areas A1 and A2 are highlighting the increased magnetic field of the entire northern half of the area. South of this border, the magnetic field strength decreases differentiating a region with relatively low geomagnetic field. One possible hypothesis that explains this observation is the increased magnetic properties of the soil and the clay layer in the northern part and the shallowing of 
limestone and carbonate inclusions in the southern parts of the area. This hypothesis corresponds to the ERT results where this zone indicates presence of more limestone inclusions in the clayed medium.

\section{CONCLUSION}

The use of two high-resolution geophysical methods - electrical resistivity tomography and geomagnetic method proved to be very effective in the non-destructive testing and survey of solid limestone inclusions in opencast Troyanovo-3 (Mini Maritsa Iztok EAD). To increase the level of confidence in the results additional studies are necessary to be performed in order to determine whether the results overlap with those of drilling. In case of confirming, geophysical methods can be used as preliminary step in carrying out studies to determine the location of the zones of solid inclusions.

\section{REFERENCES}

[1] Drahor, M.G., Integrated geophysical studies in the upper part of Sardis archaeological site, Turkey: Journal of Applied Geophysics, 59 (3), 205-223, 2006.

[2] Yunus, L. E., Kaya, M. A., 3D resistivity imaging of buried tombs at the Parion necropolis (NW Turkey), Journal of the Balkan geophysical society, Vol. 10, No. 2, p. 18, 2007.

[3] Hemeda, S., Electrical Resistance Tomography (ERT) Subsurface Imaging for Nondestructive Testing and Survey in Historical Buildings Preservation , Australian Journal of Basic and Applied Sciences, 7(1): 344-357, ISSN 1991-8178, 2013.

[4] Yigit, P., Archaeological geophysics investigation of Amorium Ancient City: Master thesis, Institute of Science and Technology, Çanakkale Onsekiz Mart University (in Turkish), 2005.

[5] Dogan, M., Papamarinopoulus, S., Geoelectric prospection of a city wall by multielectrode resistivity image survey at the prehistoric Site of Asea (Southern Greece): Archaeological Prospection, 10, 241-248, 2003.

[6] Griffiths, D.H., Barker, R.D., "Two Dimensional Resistivity Imaging and Modeling in areas of Complex Geology", Journal of Applied Geophysics, (29) 211-226, 1993.

[7] DeGroot-Hedlin, C., Constable, S., Occam's inversion to generate smooth, two dimensional models form magnetotelluric data. Geophysics, 55, 1613-1624, 1990. 